\title{
Circuit
}

Musiques contemporaines

\section{Le quatuor à cordes comme lieu du désir}

\section{Serge Provost}

Volume 11, numéro 2, 2000

Le quatuor à cordes selon Schafer

URI : https://id.erudit.org/iderudit/004690ar

DOI : https://doi.org/10.7202/004690ar

Aller au sommaire du numéro

Éditeur(s)

Les Presses de l'Université de Montréal

ISSN

1183-1693 (imprimé)

1488-9692 (numérique)

Découvrir la revue

Citer cet article

Provost, S. (2000). Le quatuor à cordes comme lieu du désir. Circuit, 11(2),

59-61. https://doi.org/10.7202/004690ar d'utilisation que vous pouvez consulter en ligne.

https://apropos.erudit.org/fr/usagers/politique-dutilisation/ 


\title{
Le quatuor à cordes comme lieu du désir
}

\author{
Serge Provost
}

À l'adolescence, le quatuor à cordes m'apparaissait comme un monde réservé et ambigu. Pour moi, qui me consacrais passionnément au piano, le violon me semblait un instrument compliqué et mystérieux dont on arrivait à sortir quelque chose qu'au prix d'années d'une gymnastique éprouvante. De plus, à cette époque, l'audition des quatuors de Haydn me révéla un monde de finesse, de lumière souvent empreint de gravité et de concentration.

Il me semblait qu'on ne pouvait être que seul pour écouter cette musique.

De ce type de relation est né le sentiment d'une distance mêlée de respect et d'envie, ce qui a suscité chez moi une forme de désir refoulé, qui a resurgi plus tard à la découverte des ensembles de violes, avec leurs sonorités ambrées, et le souffle de l'archet qui donne l'impression que ces instruments sont des êtres vivants qui exhalent leurs soupirs et leurs plaintes lje pense aux Seven Tears de Dowland). Alors l'objet de mon désir s'est précisé et m'est apparu dans toute son apparente simplicité : le sens du toucher. L'archet sur les cordes: la densité, la douceur, la légèreté, le poids de la pression, qui suscite le bruissement le plus ténu, le chant le plus clair, les sonorités les plus âpres, et ce bruit, ce souffle lqui me rappelle étrangement le frottement de l'aiguille sur les microsillons des disques de vinyle) qui colore le son et témoigne de ces attouchements mystérieux de l'archet sur les cordes tendues contre le corps de l'instrument.

Les formes de tabous ou d'inhibitions envers le quatuor à cordes, n'avaient donc rien à voir avec le poids historique des œuvres qui lui ont été consacré, et encore moins avec une forme de procès pour élitisme bourgeois qu'on pourrait intenter contre ces œuvres et le répertoire de la musique de chambre en général, dont la dénomination, par ailleurs, témoigne explicitement du domaine privé qui le caractérise, et cela même en présence de quelques centaines de personnes. 


\section{Pour qui écrire un quatuor à cordes?}

Pour moi d'abord, bien sûr, et puis pour le partager avec les interprètes et leurs instruments, et avec les amateurs, ceux qui ont une inclination particulière pour ce mode d'expression.

Il m'aura fallu du temps, plusieurs années, avant de passer aux actes; le temps de la maturation de mon imaginaire, cette région plus intime, où la spéculation intellectuelle puisse agir en toute fluidité avec un système de représentation poétique plus diffus, en affleurements, constitué des traces, des sédiments de la mémoire sensorielle, et des affects qui y sont reliés.

Les grands modèles m'ont forcément impressionné, mais sans effet dissuasif, il aura fallu qu'une œuvre en particulier me saisisse pour que mon désir se transforme en obsession. II s'agit du quatuor de Luigi Nono Fragmente-stille, an diotima, qui a fait sauter le verrou, en quelque sorte, me donnant accès à ces régions réservées de mon imaginaire.

\section{Le quatuor comme lieu de réalisations}

La première approche du quatuor s'est faite de façon presque détournée, en l'intégrant dans une plus vaste formation, lors de la composition d'Églogue, le jardin des oliviers, en 1992. Une fois intégrée la mécanique du jeu des instruments, le quatuor comme médium s'est révélé d'une grande souplesse et j'ai été même un peu étonné de m'y trouver si à l'aise. La panoplie de contraintes que l'on évoque toujours en parlant de cette formation s'est en fait révélée très riche de possibilités non seulement expressives mais aussi techniques, ce qui lui donne une grande capacité d'intégration en termes de langage musical proprement dit.

Puis à l'hiver de 1993 je me suis consacré à la composition de Vents, dont le titre est emprunté à un recueil de poèmes de Saint-John Perse, commandé par le Quatuor Morency et créé au printemps de la même année. L'écriture de ce premier quatuor a eu quelque chose de la démarche initiatique, mais pas au sens hiératique du terme, il ne s'agissait pas d'entrer dans un club sélect. Mais initiation au sens spirituel et même sensuel du terme, l'entrée dans une ascèse poétique, faite d'une attention concentrée vers un objet intériorisé, qui demande une rigueur constante et, paradoxalement, une intuition toujours en éveil.

L'expérience s'est renouvelée avec Ventis-Arboris-Vocis, commandé par RadioFrance et créé à Paris par le Quatuor Arditti en janvier 1999, et magistralement repris à Ottawa dans le cadre du festival "Cordes du Futur». Cette œuvre qui m'est très chère et qui m'a demandé beaucoup de travail, m'a permis d'entrer dans une relation que je pourrais qualifier de fusionnelle avec le quatuor à cordes et ce que j'avais à lui confier; de m'investir dans cet univers de bruissements, de rumeurs, de clameurs, dans ce jeu de perspectives sonores: auprès, au loin... de respirer à même les archets... aussi bien dans la conception des paradigmes que dans leur réalisation physique anticipée au moment de l'écriture. 


\section{Le quatuor à cordes est-il un fossile... ?}

Le quatuor à cordes me semble doté d'une vitalité que j'oserais qualifier de quasi intrinsèque, du moins tant que les compositeurs pourront y trouver un terrain propice à l'expression de leur imaginaire. II me semble que si un jour le quatuor à cordes devait disparaître, sa mort coïnciderait probablement avec celle de la poésie. 